\title{
Revitalizing Face-to-Face Local Gaming Experience through Mobile Mini Games
}

\author{
Yediya Juan \\ Computer Science Program \\ Bina Nusantara University \\ Jakarta, Indonesia \\ yediya.juan@gmail.com
}

\author{
Teuku Aulia Geumpana \\ Computer Science Program \\ Bina Nusantara University \\ Jakarta, Indonesia \\ tgeumpana@binus.edu
}

\author{
Jude J.L. Martinez \\ Computer Science Program \\ Bina Nusantara University \\ Jakarta, Indonesia \\ jmartinez@binus.edu
}

\begin{abstract}
- with the increase of mobile device usage around the globe, face-to-face communication has degraded and complete prohibition is not realistic. The degradation is not to be prevented as it happens as a side effect of technology. However, balancing usage of mobile device and quality of face-to-face communication is possible. Thus, by approaching users through developing a party genre mobile game, which is not famous as a mobile game at the time, with local multiplayer focus and introducing the given game to users, it is expected that face-to-face communication can be encouraged, thus resulting in mobile party game to become a media for the encouragement. Sample mini game that is a part of party game is developed with the help of Unity game engine as a testing object in which user response is collected. As application testing take place, and target user response is collected, face-toface encouragement is proven and user response is positive towards the game. Sample game testing has effectively reintroduced the existence of nowadays rare party game in mobile platform along with encouraging face-to-face communication, shown by the enthusiasm and enjoyment that audience testers have experienced. In the end, with mobile party game can help by being a media to encourage face-to-face communication and prevent degradation of such communication, future developments of a complete party game in mobile platform is highly encouraged as overall face-to-face communication quality enhancement may be required in the future in order to mitigate degradation.
\end{abstract}

Keywords-Games, Mobile Device, Party Genre, Multiplayer, Racing, Mini Game, Face-to-face Communication, Local Network, Unity

\section{INTRODUCTION}

With the huge increase in popularity and growth of mobile devices according to Gartner, Inc. [1], it is safe to say that most people in this world own mobile devices such as tablets or smart phones. In addition, applications for various purposes have been rapidly developed and published to fill in the memory of these mobile devices. Many companies or individuals of application developer nowadays focus on developing applications that are useful in terms of user's education, productivity, entertainment and demands. One example of entertainment-categorized application, that is no doubt a growing industry nowadays, is the development of game applications.

Games and especially mobile games have been a huge part of everyday life. Gamers and the games have started to rise up in number up to the point where games are not only entertainment options for people anymore. Global acceptance, competitive events, and popularity increase have been rising for the past few years which also result in rapid development in the gaming industry and more quantity of technical skills employed to people in regards to game development.

According to a statistic posted by 148apps.biz in regards to Apple's application store metrics, active applications within the primary category of games make up for $18.47 \%$ of the whole store's application count, leaving games in the highest position in terms of quantity of active applications (App Store Metrics, 2014) [2]. This shows that in the mobile framework, specifically for apple, a huge number of game applications have been developed and published much more than applications of a different category.

Mobile games also have the trend of multiplayer, either competitive or cooperative game play through internet/cloud service, usually connected with social networking services such as Facebook or Twitter. These multiplayer features usually stays on the border of only sharing, sending or receiving in game items, support, or tokens, while real time multiplayer gaming is not a common practice.

As games are usually either individually played offline or multiplayer online with less user interactions, local connected games with real time interaction between players are quite rare and not many mobile games support local multiplayer between devices. This results in less human interaction between players which may prove to be a problem in human communication, as face to face communication and time spending, although may not be as efficient, would be a more effective choice for communication and interaction between users.

One common fact that can be seen nowadays is that people tend to focus more on his or her mobile activities including games and online interaction that comes within the device they are currently using even though they can actually have a face-to-face interaction with their colleagues. Mobile activities that hinder the communication commonly happen within the activities of mobile texting, chatting or gaming where separate focuses between each individual towards their devices are necessary. One example of reduced face-to-face 
communication quality can be found on teens as a result of a study. The study shows that: face-to-face communication quality have reduced due to the increasing rate of texting [3].

Therefore, as one of the common mobile activities, popular mobile games, either or not would it be challenging enough to train people's mind, have a tendency to keep people from having real time spending of face to face communication between peers. This becomes a problem where the improvement of quality in game technology, which is rapidly developing, slowly reduces the quality of human interaction between peers. It is not to say however, that the use of games should be prohibited as these applications have its own benefits especially for entertainment and stress relieving, as quoted from an article written at gigaom.com by Tricia Duryee [4]. Prohibition of mobile device is unrealistic, as its usage has been staying within a high quantity, which can be seen from an article written at Venture Beat by Dylan Tweney regarding a study of how high the usage quantity of mobile devices are [5]. Yet the decrease of human interaction in gaming should not be overlooked, as it may not be a huge problem but the improvement in user-to-user interaction can prove to be useful in increasing the effectiveness and positive user experience in game applications.

In turn, the research project revolves around developing a mobile game. The main genre of choice for this project would be party game which actually consists of the requirements for developing a compilation of smaller games, a mini game to be exact within this project. This mobile game development will also take the focus of local multiplayer; the distance between each player and their respective devices determines the definition of 'local' where interaction between each user of the game can still be done physically face-to-face. Thus, the working game would be playable not through the internet, but rather, through local direct connection between each player's devices which will support a connection between two to four different devices, or in other words, also represents an interaction or act of gaming between two to four players.

As for the target platform of choice, with the uprising trend of mobile environment, iOS is selected as the primary target platform, with Android being an alternative platform. The choice of mobile environment is also preferable as the problem of user-to-user communication mainly rises due to the rise of mobile usage including games instead of games within gaming consoles or personal computers. As such, developing on mobile platform, which is widely used by society nowadays as media consumption according to Heidi Cohen's article on 25 Mobile Research Charts, is preferable [6].

In terms of technical game development specifications, the project would focus on deciding the base system and game play of each mini games, creating the mobile game on Unity game engine, applying fair to good graphical interface, applying the game's networking requirements for local multiplayer support, and finally, having it deployable on apple iOS devices.

The target of the game itself would not only be game lovers or people who would spend the majority of their time on games, but to allow users that are casual gamers, which may only play games for less than two hours of their week, to be able to enjoy the game as much and experience the gaming interaction between fellow users. The age group of the target audience is however, not set to all age group. The main target audience would be within the age group of adolescence (between 13 to 19 years old) and young adult (between 20 to 39 years old), reason being the two age group covers the majority of mobile devices usage active usage according to an article by Mark Hachman from PC magazine [7]. Post-development wise, the developed game application will be tested to see if it could actually prove to be useful in encouraging face-to-face communication.

The aim of this work is to build a playable mobile game prototype that is based on a simple mini game, which acts as a mini game example for a party based game, focusing on local multiplayer to test the effectiveness of such game in social face to face gaming experience encouragement between players. The concept of the game is broad and can be based on, but not limited to: fantasy, realistic event, political process, adventure, etc. It is expected that at the end of this project a playable local multiplayer mobile game prototype is created. The game would consist of an example mini game which game play is based on an example game from the concept of mini game compilation, where games are shorter in duration, which aims to encourage interaction between players, and is not too complex for only few people to be able to enjoy. As a result of this project, the game may not be complete or final yet, meaning that future development may take place. However, the game would be playable and can be a new source of entertainment between mobile device users. With the developed game, this research project aims to determine, whether or not a small local multiplayer mini game can be utilized to encourage a better quality of face-to-face communication between mobile device users and if whether or not can a compilation of such games be an effective media to encourage face-to-face communication quality.

As the aim of this project is achieved, benefits are expected, primarily for users to be able to enjoy a local multiplayer gaming where more real time face-to-face interaction is experienced and another would be how the game can serve as a unique type or genre of game as a source of entertainment for mobile users mainly those who uses iOS operating system. In addition, as the effectiveness of mobile device based party games in encouraging face-to-face communication is determined, future research or development can determine whether to consider or to not consider developing or having mobile device based party game as a media in which face-to-face communication can be encouraged.

\section{PROBLEM ANALYSIS}

\section{A. Problem Definition}

The problem in which this research project would like to address lies in the increasing level of mobile device usage in the society. As mobile device has become an important aspect in daily communication, people tend to spend much time focusing on their mobile devices, as shown in the result of a 
study that was led by Scott Campbell, an assistant professor of Communication Studies and Nojin Kwak, an associate professor of Communication Studies [8]. As a result, people tend to also act the same way in face-to-face meetings. For example, during a particular meet up event with their peers, people tend to stay focused on their mobile devices for the whole event due to texting, messaging, gaming, or checking their social media and in the end, the quality of face-to-face communication between the peers in that particular event degrades.

The degradation of face-to-face communication caused by mobile devices is not to an extreme extent where mobile devices completely hinder communication between peers. The degradation itself can be seen as 'minor yet slowly increasing' as the aspects of hardware and software (application) in mobile devices experience rapid development. Having this degradation, however, is not preferable as face-to-face communication is essential and important in human life, which is why it is called degradation. The essentiality of face-to-face communication is due to the fact that remote communication such as texting may bring miscommunication between communicators, as written by Erwin Stewart, an editor at The Lance website [9].

Another important aspect of this problem is how it is not solvable by preventing or erasing the use of mobile devices. Again, mobile device have become an important aspect in daily life, given that it is a result of development in technology that supports the society and plays a crucial role by proving to be useful in the society's efficiency. Thus, with the huge number of users consisting of wide range of age group and ethnicity around the world, completely disabling access to mobile devices is just not a viable or realistic option.

\section{B. Proposed Solution}

The problem of degradation in face-to-face communication is not solvable by complete prevention in mobile device usage as it is unrealistic. However, as the problem should not be overlooked, it is viable to maintain mobile device usage instead of isolating it. There comes the reason for the proposed solution, which is to develop a mobile party game with the aim of encouraging face-to-face communication. The encouragement aimed by this research project is not meant to completely stop the degradation; it is however, to maintain balance in mobile device usage and face-to-face communication between peers by providing an entertainment using the mobile device itself in the form of a game application. In short, the approach taken by the authors in maintaining the problem is by developing a mobile party game prototype and determines the game's effectiveness in encouraging face-toface communication.

There are several reasons of why the given approach for the solution is chosen.

1) Developing a game application for mobile is chosen because a game is a form of art and entertainment. Games have already been a common entertainment form in mobile devices. Therefore, users would be familiar and be able to adapt quickly with the developed application. Game also provides fun and challenge at the same time; this means that it is an interesting form of entertainment that would be acceptable by users. Also, mobile platform is chosen because the quantity of mobile phone users has increased and that allows more people to be able to experience the game application.

2) As the problem lies in the degradation of face-to-face communication, the game genre of choice should address this problem, which is to improve communication. Therefore, multiplayer option is preferable. Also, the game should be able to take the interest of wide range of audience who use mobile phones; and mini game is a potential choice, as it would allow audience to be able to enjoy the game easier without having to learn too many deep aspects. In order to help improve communication, the competitiveness of the game should be set to mild and this could also be found on simple mini games. Thus given these conditions, party genre fits the criteria well, since it focuses on multiplayer and compiles several mini games into one whole game.

3) Currently, many party games have been published around the globe, mostly available for game consoles such as PlayStation or Nintendo Wii. These console party games are built based on a compilation of mini games. As for mobile platform however, search results in Apple app store shows that no party games, which consist of several mini games, have yet to exist. So far, the only existence of party games application on mobile devices is tools to help in classic games when people gather, instead of actually mobile video game. Thus, the application available on mobile is not a game application, but more of a tool application used in exchange of paper to help gatherings.

4) Several notable examples of current existing party games and mini games include:

a) Crash Bash, a once famous party video game made for Sony PlayStation on the year of 2000 featuring a bandicoot called Crash [10]. The game consists of multiple smaller mini games with playtime ranging from around 2 to 3 minutes. In this game, players can play against one another in versus mode, or work together to finish the story mode. Although the game consists of smaller mini games, a story mode which includes a storyline is available so that players would have to progressively finish several mini games in order to unlock more advanced mini games and proceed through the storyline.

b) Pac-man Fever is another party game featuring the famous classic Pac-man character together with several NAMCO characters such as Heihachi from the game Tekken, supported by a review written at gamespot.com [11]. In 2002, the characters are remade in 3D graphics and are placed in a party genre setting. Like most party games, this game is composed of many different mini games, approximately 30 to be precise. One unique aspect of this game, however, is how players will compete in a tiled board race, similar to a game of Snake and Ladders. Every player is placed in the starting tile, and whoever reached the finish tile first is the winner of the game. Every turn in this game, 
all players will enter a 4 player mini game and how good each player performs in the mini game will determine how many tiles he or she will move. Thus, instead of rolling dice, a player can only move farther and reach the goal faster than other players by being the top player in every mini game.

c) Mario Party features the famous Nintendo character, Mario. Initially, the very first Mario game was not made as a party genre game. However, as time goes by and Mario gains popularity, many spin-off and side story games are created, one of it being the Mario Party series. The first Mario Party was announced and released on 1998 in Japan for the console Nintendo 64 and is playable for up to 4 players, according to an article by Peer Schneider at ign.com [12]. Each player can choose a character that is included in Nintendo's Mario franchise such as his brother and sidekick Luigi or the antagonist Bowser. Up to the moment of this thesis's writing, the Mario party series have reached the 12th game and is currently working on the 13th, showing that many Mario party series have actually been publish and is being played around the globe. Each sequel of the game doesn't always follow the exact mini game style or storyline of the former, but as the game proceeds, the base stays approximately the same.

d) The game Pokemon Stadium, is not actually a party genre game as it more of a Fighting Strategy game. However, there is a party game feature built within the game. When the main player moves around the main menu, which is interfaced in the form of a map, he or she can pick an area called the Kids Club, where a side party game is located. This mode has several mini-games which supports multiplayer. The party game within this game features Pokemon characters that are also owned by Nintendo Company and is the main franchise character of the game itself, as can be seen from the mini game list in Pokemon Stadium given by a Pokemon website called Psypokes [13]. The aspect of having a party game built inside a whole different game like Pokemon Stadium is unique in games and is not often found in many games.

Table 1 shows that all current existing party games, only exists in video gaming consoles such as Sony PlayStation or Nintendo Wii. Thus, the first advantage of the proposed solution is the portability of the game. This became an advantage due to the fame and width of mobile phone usage, and the device availability itself towards wide range of users. As the number of mobile device users increases, games based on any mobile device gains great advantage against home console games due to the availability of audience and users. However, as the proposed solution's main intention is to collect the response of users towards the existence mobile party game, the application itself will not be a complete application. Thus, storyline and a base board will not be available. Depending on the testing results, however, futures developments of the game may, and will most probably, include a base board and storyline to increase the appeal of the game, as this is a famous feature implemented by console party games. Yet possibly, the board and story mode implemented in the proposed solution mobile game will be in smaller scale, as the game is played on mobile platform and longer games may not be preferable.

TABLE 1. EXISTING GAMES AND PROPOSED SOLUTION COMPARISON

\begin{tabular}{|l|l|l|c|}
\hline \multirow{2}{*}{\multicolumn{1}{|c|}{ Game Title }} & \multicolumn{3}{c|}{ Comparison Table } \\
\cline { 2 - 4 } & \multicolumn{1}{|c|}{ Genre } & Platform & $\begin{array}{c}\text { Progressive Story } \\
\text { Mode }\end{array}$ \\
\hline Crash Bash & Party & Game Console & Available \\
\hline PacMan Fever & Party & Game Console & Unavailable \\
\hline Mario Party & Party & Game Console & Available \\
\hline Pokemon Stadium & Non-Party & Game Console & Unavailable \\
\hline Proposed Solution & Party & Mobile & Undetermined \\
\hline
\end{tabular}

\section{Pre-Development Survey}

Before development of the proposed solution is developed, a pre-development survey has been conducted. The survey is conducted by publishing an online questionnaire and sharing the link through social media websites, mainly on Facebook. The main purpose of conducting the survey is of course, to gain response from public regarding the proposed idea and solution. The questions included in the survey mainly revolves around knowing the respondent's usage of mobile device, their familiarization with mobile games, their familiarization toward party genre games, and their opinion on mobile party games.

From the conducted survey results of 29 respondents, most respondents are within the age group of $20-25$ years old which covers $64 \%$. In terms of mobile device usage, many of the respondents are currently using either an iOS based phone, or an Android based phone, $48 \%$ and 38\% respectively. This shows that every respondent owns at least one mobile device. The usage time of each respondent's mobile device is also quite high, with most of them, specifically $78 \%$, spending around 1 to 15 hours per day with their mobile device. This also shows that their ownership of mobile device is active, which means that respondents actually own and use the device in their daily life instead of just owning and not using it. From these results, it can be concluded that people actually do spend hours of their time per day for their mobile device usage. 93\% of the respondents, which actual number is 27 people, responded that they have played mobile game at least once in their life. Most respondents have their favorite game genre as Puzzle genre, followed by Strategy, and Adventure. Party became the favorite, only for one respondent. However, the reason can be seen from the result of the next question, which is whether or not do they know about what a party game is. For this question, $55 \%$ of the respondents answered that they do not know about the genre, whilst the remaining $45 \%$ either knowing about the genre well or only know about the genre roughly. After the definition of party game is given, however, it is shown that $72 \%$ of the respondents have actually played the game, despite not knowing the genre definition. This may show that the knowledge regarding party game is not really popular possibly 
due to the game is known more as a mere multiplayer mini game. Due to such, Party genre game, which is not really famous for now, can be reintroduced as a new experience for mobile entertainment. After questions regarding mobile device usage is enquired from the respondents, the next question focus more on their opinion on daily communication. When asked regarding whether or not face-to-face communication have degraded between peers due to the existence of mobile devices, most of the respondents answered that face-to-face communication really have degraded. $48 \%$ answered that it really have degraded, followed by $28 \%$ answered that it have "degraded by a bit", which means that degradation happens in the slightest. Thus, it can be seen that face-to-face communication really has degraded, although only slightly. As the authors tried to ask the respondents' opinion regarding whether or not mobile games can be a media to encourage faceto-face communication, $69 \%$, which is 20 , of the respondents thought that mobile games can be used as a media to encourage face-to-face communication. Finally, the last question asks about whether or not the idea of developing a mobile party game would be interesting for respondents. As a result, 52\%, which means the majority of respondents are interested and it can be concluded that development of a mobile party game is a viable solution to encourage face-to-face communication.

To sum it up, as a conclusion from pre-development survey, with wide usage of mobile device which is still increasing (due to rapid development of technology) and majority's familiarity with mobile games, mobile game development has good prospect in terms of usage. Further, as face-to-face communication has degraded and games can be a media to mitigate and balance the degradation, it is decided that development of the proposed mobile game application should take place.

\section{SOLUTION DESIGN}

\section{A. Solution Approach}

In developing this project, the software development technique that will be implemented is the Software Development Life Cycle Methodology, specifically using Incremental - Iterative Model Approach [18]. In development of this research project, it is planned that around two to four iterations of development for this application should take place. The exact number of iteration however, would depend on the progress of the application after each iteration. As soon as the application has no error that could hinder user acceptance testing, which is deployment, no more iterations will be undergone and the application will be taken to deployment phase. The planning of what will be done during each steps of the Incremental - Iterative Model Approach, which is the approach of how the project will be initiated, is given below:

\section{1)Initial Planning}

During this phase, the scope and aim of this project will be defined and established. As this phase is not included in the main iteration of the project, this phase will only take place once. The planning would then produce a decision of what would generally be created in this research project, this decision will then be the base in which other phases take place.

\section{2)Planning - Requirements Elicitation}

Planning, as the second step of the development, is the first step in the main life cycle iteration. During this phase in the very first iteration, the first planning of details on what would be made in this project will be established. Thus, establishment of the requirements on what should be included and what should the expected result be after development take place. This may include planning on what detailed features would be included in the application. After the first iteration however, this phase will discuss about what changes should be made and how should it be applied after evaluating the former development result.

\section{3) Analysis and Design - Implementation}

During this phase, requirements established during the planning phase are applied. Programming and development of the project will take place within unity. The result of this phase should be an application which has functionalities defined in the given requirements.

4) Testing

The testing step is again, simply testing the developed application. To be precise, the testing that will take place during this phase is the alpha test, which would be the testing that is conducted by the author.

\section{5) Evaluation}

During the evaluation phase, every testing phase notes and results will be evaluated and defined in order for the next planning to take place. This phase would also include the decision making of whether or not any results of testing should be taken for update in the next iteration. Up to this phase, iterations can take place several times before commencing into deployment phase.

\section{6)Deployment}

In this phase, the final product of the application will be taken for User Acceptance Test. The test includes 6 defined key aspects or features for utilization to determine the game's effectiveness in encouraging face-to-face communication. The 6 aspects are: Racing Game Play, Characters, Speeding up Mechanism, Slowing down opponent(s), Multiplayer support, and graphic design.

\section{B. Application Specification}

For the application development, some detailed specification of the application has been established and are given as follows:

1) The application built will be intended for mobile platform.

2)During this research project, a full application may not be made as the purpose of this project is to evaluate the effectiveness of mobile party game by providing some party game examples to testers and audience.

3) The application will be built in two-dimensional style graphics.

4) The application may not have all complete functionalities, but will have its core functionalities set.

5) The main target audience of this application would be around the age group of adolescence (between 13 to 19 years old) and young adult (between 20 to 39 years old). 
However, other audience should be able to also use the application.

6) The application will have networking support, built in by the game engine, in order to allow multiplayer features.

7) Although a complete set of mini games is preferred, due to the existing time limitation for development, the mini game created for this application is a racing game application. This is because racing is a simple and commonly known gameplay that people are familiar with. In addition, racing gameplay is also sufficient to achieve the aim of this research.

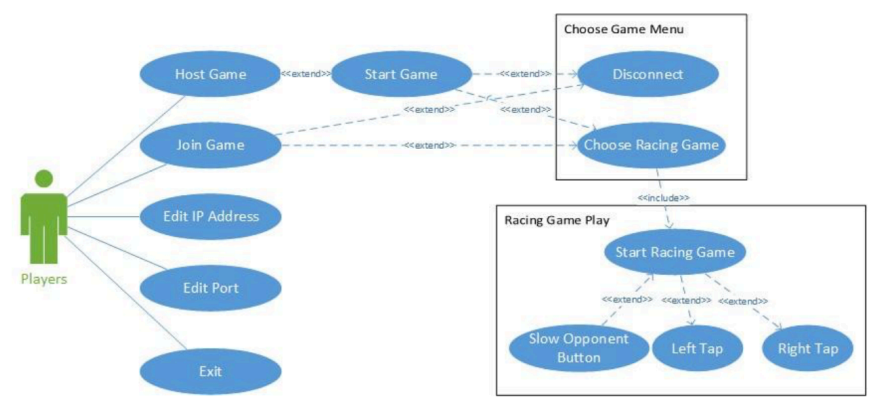

Figure 1: Racing game use case diagram

\section{SOLUTION IMPLEMENTATION}

\section{A. Initial Planning}

During the initial planning, the overview of the whole project is first determined. This includes the determination of the background of the problem, the scope in which the development will overtake, and the aims and benefits of the development itself. Next, the timeline of the development is also decided. In this case, the timeline would be during the duration of this thesis, which is around four months long. Also, each step, progress and documentation that has to be created for the whole project to finish is defined. In this case, the document is the thesis book itself, and the completion of development is the game application for testing and receiving user response of does the application encourage face-to-face communication or does it not. In addition, pre-development survey is conducted, in order to receive response from public users and audience regarding the beginning of the project. The result of the survey is then also used as a base motivation of the aim of the project. Then, the main problem in discussion is also determined, in order to have a clear sight on the focus that should be achieved by this application, which is to, again, determine whether or not face-to-face communication is encouraged by party games.

\section{B. Planning-Requirements Elicitation}

During this step, in the first iteration, the details of the requirements of the project are defined. These details include the specification of the application given in chapter 4. Also, planning on how the game should be designed take place, as the purpose of the game is not only for entertainment, rather in this project, more for research purpose. To sum it up, the requirements are: for the game to support local multiplayer, multiplayer can include up to 4 players, and for the game to be able to be used as a test media in which tester's opinion on their face-to-face communication in playing this game is received. During the following iterations, however, planning is more in detailed as in the modifying or fixing of any errors and bugs found after testing phase. This focus on what errors to fix and what is considered to be fixed.

During the second iteration, planning is focused more on the case of networking and point coordination converting. In terms of networking, it is planned that network object would no longer be created on each different scenes as it generates error and network multiplication which cause the application to crash. Rather, network will be applied on a single scene, which is the main scene, and will be passed along to other scenes. Thus, no multiple copies of the network object would exist at the same time. In terms of point coordination, it is decided that point coordination should only be applied for graphical user interface objects such as buttons and text input boxes, as these objects would dynamically follow the screen of the game. Other objects however, do not need to have their coordinates converted as the placement of these objects can be left static whereas the placement of the camera is modified to center at any of the intended static objects. Thus, animation and movement effects can take place by moving the camera instead of converting object points and moving them.

\section{Analysis and Design-Implementation}

During this step, the implementation and development take place. According to the planned steps on the former step, the application should have specifications and details in architecture as explained in application modeling in chapter 4 . During implementation, some problems take place, mostly bugs and errors that the author is not familiar with, due to using a game engine that the author has not mastered yet. The upside in the development is that, Unity game engine is well known and many frequently asked questions, support forums, and tutorial videos or articles are widely available. Thus, in order to overcome the problems, the author seeks help and support which came in the form of recommendation and possible fixes of similar problems from forums or the programming language API documentation.

One main problem in the first iteration within the implementation phase is during implementation of networking, as the logic of network connection in hosting open connectivity rooms and joining them may sometime confuse developers. Other than network problem, character movement and asynchronous movement between different devices while having information sent simultaneously is still a problem. However, with simultaneous programming and testing, replanning can take place and problems of the sort can be mitigated.

During the second iteration, network problems are addressed and the generation of network objects is modified such that it does not generate any further error. Also, object coordinate is set to static and instead, camera coordinates are modified. 


\section{Testing}

In this step, the testing that took place is not user testing. Instead, the testing that takes place is the testing done by the author. The designer that also focus on the design of this game also does some testing to see which bug or error appears and how should it actually behave. Thus, after testing the application, the author is able to grasp the condition and faults that happen within the game.

During the first iteration, it is found that the network object clash with each other and players of different devices or copy of the application is unable to see the remote player's character in their device. Also, after objects are placed and coordinates of the objects are established, scripts that intended to take the object coordinates and use them to generate dynamic modification during game play fails to respond. As a result, some game objects are placed farther than where they should actually be.

The second iteration however, finds that problems showing on the first iteration are addressed.

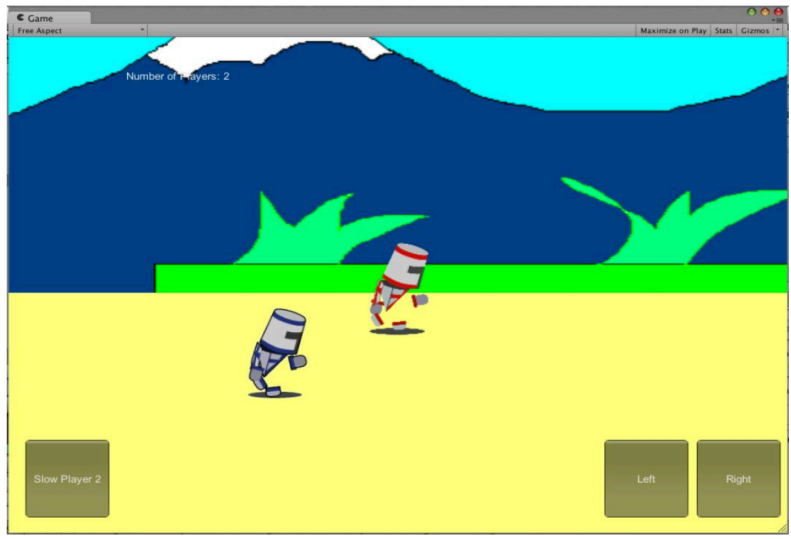

Figure 2: Racing Game Screenshot

\section{E. Evaluation}

In this phase, as the author have known about which behavior is faulty and require re-programming and bug fixing, the author evaluates and re-check the codes in order to look for the source of logic error. Due to the compiler's ability, fortunately, syntax error can be easily mitigated. Logic error, however, require more iteration of re-planning, reimplementation, and re-testing before re-evaluating the codes once more. Most logic errors happen due to the misusage of any API functions of the programming language.

For example, some network functions have the name and documentation that implies that defining and calling the function would automatically create an established network which would only require less settings. However, that is not the case, as the network has to be bind to a port. This doesn't mean that how it works is more complicated. However, the authors at times, fail to understand how the function actually works. But with more learning, this problem can also be mitigated.

\section{F. Deployment}

In this project, as stated in the project scope, the deployment is not meant to be a deployment of complete program. Instead, the deployment is the conduct of user acceptance testing, and collecting the tester's opinion and feedback for the use of future developments and determination of whether does party games (or the given mini game) is an effective media to encourage face-to-face communication.

\section{G. User Acceptance Testing}

After development takes place and application is ready for beta testing, the game application which consists of one example party game, which is the racing game, undergo beta testing. This beta testing is called User Acceptance Testing, which main purpose is to see if the application is accepted by target audience and the public. This phase can be considered as the final phase, or also called as the deployment phase of this project. This is due to the purpose of the research project that is to determine whether or not does a party game application is effective as a media in which face-to-face communication can be encouraged. The testing is conducted by collecting several volunteer respondents, asking them to test and play the game, and have them answer a questionnaire prepared by the author. The questionnaire itself is designed by the author so that the answer could be concluded to determine whether or not the application is accepted by public users, and whether or not faceto-face communication between users is encouraged. This section will focus on showing the results of the user acceptance testing, along with the brief conclusion of that test.

In the conducted test, 27 respondents are asked to be a volunteer in testing this application. Out of those 27 respondents, the main age group of the testers, taking up 52\%, is from the age of $20-25$ which would be the initial target audience of the application, followed by $26 \%$ from the age group of 14-19, and 26 - 30 with only 3 respondents. Respondents in age group of below 14 and over 30 do not have that many people taken as testers, as the main target audience of the application is not these age groups, yet having feedback and response from them can support in some facts.

Of the 27 respondents, all of them specified that they have played mobile games in their life before, at least once. And thus, the game application is initiated in order for them to test. During testing, at least a pair of respondent is asked to play the game together as the application requires multiplayer usage. If there only one person at one time is available for conducting the test, then multiplayer testing is done with the help of the author. The author however, is not included in the survey result of the testing within this case.

After testing, $81 \%$ of the respondents answered that they can understand the interface and use of buttons easily within the game. The remaining 19\% however, does not. This may be the result of using a networking term in the game such as host game, IP address, port, and join game. Although the terminologies have been quite famous, especially in those that are familiar with games, some people who are not familiar with games may find these terms not understandable. In addition, $52 \%$ of the respondents stated that the game room hosting and 
joining is efficient; while $48 \%$ other does not. A feature that can be implemented in mitigating this may include easier room creating and joining, instead of the requirement for IP address and port, having a list of rooms hosted in the interface, in which the rooms can be found public without the need of typing IP address of the target device. In terms of joining or leaving game room, 6 respondents find it hard to do this, mostly due to the inability of the device to connect to the given IP address or blocked port number. 21 other respondents do not encounter any problem in joining game rooms.

The question then starts to focus on the game aspect. When asked about whether or not is the game interesting for the testers, $85 \%$ replied that the Racing Game is an interesting example game. This may be due to the example game being simple and have a unique control in accelerating and decelerating characters. The remaining $15 \%$ does not find racing game to be that interesting. However, when asked regarding the fun aspect of the game, $89 \%$ responded that the game is actually fun to play. This adds up to the fact that although the game may not seem interesting, having fun during multiplayer game is still possible. Also, $81 \%$ find that the racing game isn't hard to play. The remaining $19 \%$ however, find it quite hard to control the alternative left-right tapping and in winning the game by out speeding their opponent in the alternative tapping.

In this game as stated before, 6 key aspects are used to evaluate and are defined as the following: Racing Game Play, Characters, Speeding up Mechanism, Slowing down opponent(s), Multiplayer support, and graphic design. In terms of which one of the aspects is most appealing, majority of the respondents answered: Speeding up Mechanism and slowing down opponent(s), which counts for $37 \%$ and $33 \%$ respectively, followed by the multiplayer support aspect by 4 respondents and characters aspect by 3 respondents. Only one respondent, answered with "racing game play".

In terms of which one of the aspects is the least or even not appealing and is disliked, majority dislikes the graphic design, which counts for $63 \%$. This is most probably due to the designs being a temporary poor dummy design prepared by the author as the only design ready by the designer and is implemented in the game already, is the character design. The answer of graphic design however, as asked from the respondents, does not include the background interface for the main host game menus.

Thus comes the most important and main target of the research project. The question asked was whether or not the game encourages face-to-face communication between each player. And $92 \%$ of the respondents answered with "Yes". This actually shows that mini games with simple rulings can actually be used as a media to encourage face-to-face communication. Then, it is also asked for their opinion, of which aspect from the 6 features or aspects would be the one to encourage interaction with friends. For this question, the answer given is Multiplayer Support, which is the answer of 11 respondents, being $41 \%$. Other remaining respondents answered Racing Game play, Speeding up Mechanism, and slowing down opponent(s). The remaining 2 aspects are not picked by any of the respondents.
From here, it can be concluded that the encouragement comes mainly from multiplayer support, as the respondents are able to communicate directly with their opponent(s), followed by the type of game that is played. Specifically, the multiplayer support that is given should be local multiplayer (in which players can communicate face-to-face), in contrast to most online games now which focuses on remote multiplayer. Then, the type of game played should be simple, have unique mechanism that can annoy or cheer the players and their opponents. With these two combinations of simple gameplay and local multiplayer, players are able to focus their attention more on between-player interaction, usually regarding what they are experiencing in the game, and less on the game itself.

Finally, questions are asked of whether or not the respondents would be interested to play the game with their friends if more mini games are implemented, in which $93 \%$ of the respondents answered as they would. In terms of preference, 24 of the respondents would prefer to play against their friends instead of a computer AI, with the reverse answer for the remaining.

To strengthen conclusion, the respondents are asked from a scale of 1 to 5, of do they think that the game can be a media to encourage face-to-face communication, and a point of 4 and 5 is answered by a total of $70 \%$ of the respondents, while the remaining being neutral or does not think that the game can be a media to encourage face-to-face communication. However, no respondent answered ' 1 '. In terms of scaling their interest for the mini game application itself, again majority, being $48 \%$, answered with yes, whilst the other $30 \%$ answered with neutral. One respondent answered ' 5 ' while the remaining answered ' 2 '. Again, no respondent answered ' 1 '. In the end, the satisfaction of the testers is $89 \%$ satisfied and the remaining unsatisfied with the game application.

Several feedbacks, critiques, opinions, and recommendations are also asked from the testers. Many of them do not have any recommendation for the game. Yet some recommendations revolve around fixing the graphic design of interface, improving the means for device connection, and adding more games. A room chat feature is also one of the recommendations, and another recommendation includes critique in which there are too much future developments due to dummy poor graphics and only one example game.

\section{CONCLUSION}

To conclude this research project, as it can be seen from previous sections that the background of which the quantity of mobile usage have increased and the quality of faceto-face communication degrades. Thus, the solution of developing an example party game is proposed and implemented for testing of whether or not the game can be used as a media for face-to-face communication encouragement, although the solution itself is a game. The results, however, is interesting as after testing results are collected, it can be concluded that face-to-face communication is really encouraged with the game. Also, the use of the game in mobile device can be implemented well especially in iOS as it is the main target platform in which the game is built. Also, the 
buttons are made for touch screen devices, which is the majority of mobile device nowadays. This also supports mobile device implementation. Thus, to sum it up, future development of party games in mobile device could be done for the purpose of encouraging face-to-face communication, which has been degrading, between mobile device users.

\section{REFERENCES}

1. Gartner Says Worldwide Traditional PC, Tablet, Ultramobile and Mobile Phone Shipments On Pace to Grow 7.6 Percent in 2014. (2014), Retreived June 12, 2014, from http://www.gartner.com/newsroom/id/2645115

2. App Store Metrics. (2014). Retrieved April 24, 2014, from http://148apps.biz/app-store-metrics/? mpage $=$ appcount

3. Teens Prefer to Communicate via text --Study. (n.d.). Retrieved April 24, 2014, from http://www.guidetoschools.ca/blog/Calgary-SchoolsTeachers-Parents-Teens-Prefer-to-Communicate-Via-Texting-Study

4. Report: Mobile Games used As Stress Relief, Often Played At Work. (2009). Retrieved May 4, 2014, from http://gigaom.com/2009/05/06/419report-mobile-games-used-as-stress-relief-often-played-at-work/

5. Mobile App Growth Exploding, and shows no sign of letting up. (2013). Retrieved May 3, 2014, from http://venturebeat.com/2013/07/10/state-ofthe-appospherel

6. 25 Mobile Research Charts to Guide your 2012 Marketing. (2012). Retrieved June 2, 2014, from http://heidicohen.com/why-you-needmobile-marketing-now-research/

7. Majority of Americans Now Own Smartphones, Survey Says. (2012). Retrieved June 12, 2014, from http://www.pcmag.com/article2/0,2817,2404086,00.asp

8. Study Discovers how cell phone use affects social interactions. (2011). Retrieved June April 20, 2014, from http://www.michigandaily.com/news/'u'-researchers-identify-linkbetween-cell-phones-and-socialization-habits

9. Does cell phone use really affect our communication skills? (2013). Retrieved June 10, 2014, from http://lhslance.org/2013/features/cellphone-use-really-affect-communication-skills/

10. Crash Bash Review. (2000). Retrieved April 9, 2014, from http://www.gamespot.com/reviews/crash-bash-review/1900-2651252/

11. First Look: Pac-Man Fever. (2002). Retrieved April 9, 2014, http://www.gamespot.com/articles/first-look-pac-man-fever/11002866112/

12. Mario Party.(1999).Retrieved April 9, 2014, from http://www.ign.com/articles/1999/02/12/mario-party

13. Pokemon Stadium 2 Mini Games. (n.d.). Retrieved June 15, 2014, from http://www.psypokes.com/stadium2/minigames.php

14. What is a Game engine?. (2008). Retrieved April 23, 2014, from http://www.gamecareerguide.com/features/529/what_is_a_game.php

15. Apple iOS. (n.d.). Retrieved May 10, 2014, from http://www.webopedia.com/TERM/A/apple_ios.html

16. Game Genres. (n.d.). Retrieved April 26, 2014 from http://www.allgame.com/genres.php

17. Definition of a Party Game. (2012). Retrieved June 10, 2014, from http://www.saratogafalcon.org/content/definition-party-game

18. Agile Development. (n.d.). Retrieved April 1, 2014, from http://agiledevelopment-tools.com/

19. A Comparison Between Three SDLC Models Waterfall Model, Spiral Model, and Incremental/Iterative Model. (2015). IJCSI International Journal of Computer Science Issues, Volume 12, Issue 1, No 1. Retrieved December 10, 2016, from http://www.ijcsi.org/ 